\title{
Classification in Child and Adolescent Psychiatry: A Clinician's Perspective
}

\author{
Devashish Konar MD Consultant Psychiatrist Mental Health Care Centre Kolkata
}

Do we need a diagnostic tag all the time? For communication in clinical practice, research, public health planning, the legal system, insurance reimbursement, and disability evaluation, we do need them. In the case of clinics, a good formulation may be more useful. While trying to classify we cannot afford to forget that society has great concern if you label the child or adolescent inappropriately.

Diagnostic and Statistical Manual of Mental Disorders, $5^{\text {th }}$ Edition (DSM-5), Diagnostic Classification of Mental Health and Developmental Disorders of Infancy and Early Childhood (DC:0-5) and Research Domain Criteria (RDoC) are already in use and International Classification of Disease, $11^{\text {th }}$ Revision (ICD-11) is in late gestation. Labeling a child or adolescent with a diagnosis is a sensitive issue and understanding the finer nuances is a must for clinicians. Across the classification systems, the effort is to understand the illnesses neurobiologically, add dimensions in addition to categories whenever possible, and to make the description more narrative and culturally sensitive.

\section{Changes brought in DSM-5}

DSM-5 has tried to combine dimensions and categories, has put an effort to make it neurobiologically informed, and has promoted transition to a life span perspective.

Autism Spectrum Disorder has simplified things, prevalence of bipolar disorder is restricted by creation of new category Disruptive Mood Dysregulation Disorder, diagnosis of PTSD is easier in early childhood and ADHD requires the presence of symptoms in multiple environments.

Multiaxial system has been discarded in favour of more narrative description. Critical cultural considerations have added refinement to it.

\section{What is new in DC:0-5}

ZERO TO THREE got its recent revision in 2016 to DC:0-5. Update criteria for classification, introduces several new disorders, expands the age range from birth through 3 years old to birth through 5 years old, continues with the multiaxial classification system, substantially revises the axes.

Axis I: Clinical Disorders

Axis II: Relational Context

Axis III: Physical Health Conditions and Considerations

Axis IV: Psychosocial Stressors

Axis V: Developmental Competence

\section{RDoC by NIMH}

The second time in 40 years after RDC of 1970s, in RDoC the spot light is on the validity of diagnostic system, based on the framework for research into the biology of mental illness. The new approach is working on markers of underlying mechanisms such as measures of cognition, brain structure, and neurophysiology. Is R-DoC the answer ? Clinicians and researchers need to work in harmony.

\section{Changes expected in ICD-11}

$70 \%$ of the mental health professionals around the world use ICD-10 for making psychiatric diagnoses in clinical settings. So a major shift in clinical data keeping is envisaged with ICD11, which is expected to begin on January 1, 2022. The whole process of development of the manual has ensured a greater openness and has been more responsive to differing viewpoints in the realms of behavioural sciences.

Largely successful efforts have been made to harmonize the ICD 11 and DSM 5 classification systems. Cultural issues have been taken care of. There has been a major focus upon a manual for primary care.

It would be interesting to understand how the four systems' approaches (DSM-5, DC:0-5, RDoC and ICD-11) to the key issues of diagnosis correspond or diverge as a result of their different histories, purposes, and areas of implementation. Although the systems have varying degree of overlap and distinguishing features, they should share the common goal of reducing the burden of suffering due to mental disorder and should facilitate communication between the stake holders.

The common misconception of a beginner would be that all individuals under the same diagnostic category would be alike in all important ways. With clinical maturity you learn that for people with the same mental disorder, defining features being the same, may well differ in many other important ways that may affect their clinical management and outcome. 